\title{
A case of bilateral forearm amputation resulting from purpura fulminans following subtotal colectomy for perforated ulcerative colitis
}

\author{
Jialing Zhu*, Shivali Mukerji and Ala Nozari \\ Department of Anesthesiology, Boston Medical Center, USA
}

\begin{abstract}
Introduction: Purpura Fulminans $(\mathrm{PF})$ is a complication commonly associated with scarlet fever and meningococcal infection in infants but is also reported in adult patients. Limb ischemia is a devastating complication and amputation may be necessary to reduce mortality.

Case report: We report a 49-year-old, previously healthy female patient, who presented with ulcerative colitis and underwent a colonoscopy. Post-operatively, she presented one week later with transverse colon perforation requiring subtotal colectomy, abdominal washout and ileostomy. Her case was further complicated by an intra-abdominal infection causing septic shock. She then developed purpuric skin patches, sparing the trunk, and severe ischemia of her distal extremities consistent with PF. Despite improved respiratory and hemodynamic status, her limb ischemia continued to progress, and she eventually underwent bilateral forearm amputations.

Discussion and conclusion: This case presents an opportunity to discuss the importance of supportive care and hematological treatments to reduce the mortality and control the devastating complications of microvascular coagulation and limb ischemia associated with PF. Optimization of microcirculation, steroid treatment, immunomodulation, protein $\mathrm{C}$ zymogen, plasma exchange or replacement can potentially mitigate the injury and should be considered to improve outcome.
\end{abstract}

\section{Introduction}

Purpura fulminans $(\mathrm{PF})$ is a rare syndrome characterized by microvasculature coagulation and thrombosis with hemorrhagic necrosis that can lead to purpuric lesions, skin necrosis and gangrene and autoamputation of distal extremities [1]. It has been reported as a complication to scarlet fever and meningococcal infections in infants and young children and is associated with acquired or congenital Protein $\mathrm{C}$ and $\mathrm{S}$ deficiencies [2,3]. Although less common, PF has also been reported as an idiopathic disease in adults with protein $\mathrm{S}$ deficiency and also as an acute infectious form in patients with sepsis and disseminated intravascular coagulation (DIC) [4]. We report a case of a 49-year-old female with ulcerative colitis who developed PF with limb ischemia and gangrene as a complication to transverse colon perforation and sepsis and discuss treatment strategies that are likely to improve the outcome.

\section{Case report}

A 49-year-old previously healthy female presented with hematochezia and underwent a colonoscopy with diagnostic findings of ulcerative colitis. She was discharged to her home with prednisone taper for four weeks. The following week she presented with a transverse colon perforation, requiring subtotal colectomy, abdominal washout and ileostomy. Her postoperative course was complicated by polymicrobial intra-abdominal infection causing septic shock. Treatment with broad-spectrum antibiotics were initiated immediately and hemodynamic support with fluids and vasopressors was provided. On postoperative day 3, patient developed non-blanching, angulated, purpuric smooth skin patches, and thin plaques with areas of hemorrhagic bullae over her distal extremities, sparing the trunk. Over the next 24 hours she developed dry gangrene of bilateral upper and lower extremities.

Laboratory results revealed significant thrombocytopenia and elevated prothrombin time, partial thromboplastin time, as well as elevated D-dimer and decreased fibrinogen, consistent with DIC. Skin biopsy revealed thrombotic vasculitis and findings consistent with purpura fulminans. Bilateral upper extremities doppler ultrasound showed deep venous thrombosis.

Patient received platelet transfusion when platelet count decreased to 18,000 platelets per microliter of blood. Anticoagulation with heparin infusion was initiated as her thrombocytopenia started to resolve. Treatment with fresh frozen plasma was also started but was limited by patient's intolerance to fluid resuscitation and congestive heart failure.

Dry gangrene of bilateral upper extremities continued to progress despite resolution of her septic shock (Figures 1 and 2). Patient's hemodynamic and respiratory status gradually improved, nevertheless, and she underwent bilateral forearm amputations 72 days after her subtotal colectomy.

${ }^{*}$ Correspondence to: AJialing Zhu, Department of Anesthesiology, Boston Medical Center, USA, E-mail: Jialing.Zhu@bmc.org

Key words: purpura fulminans, forearm amputation, necrosis, gangrene, coagulopathy

Received: January 28, 2021; Accepted: February 08, 2021; Published: February 11,2021 


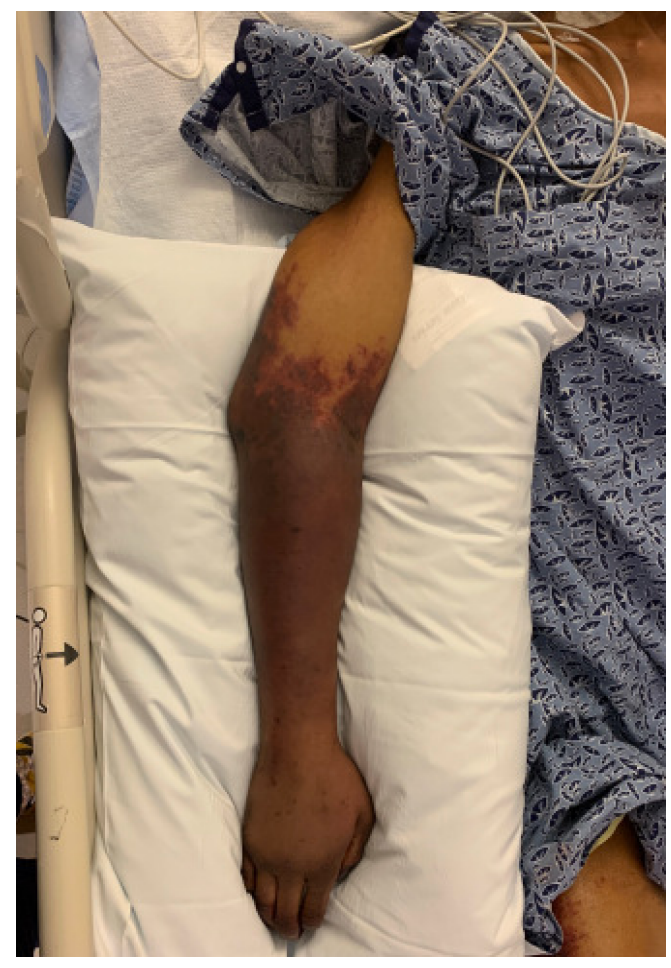

Figure 1. Right upper extremity, post-operative day four, visible demarcation of necrotic changes

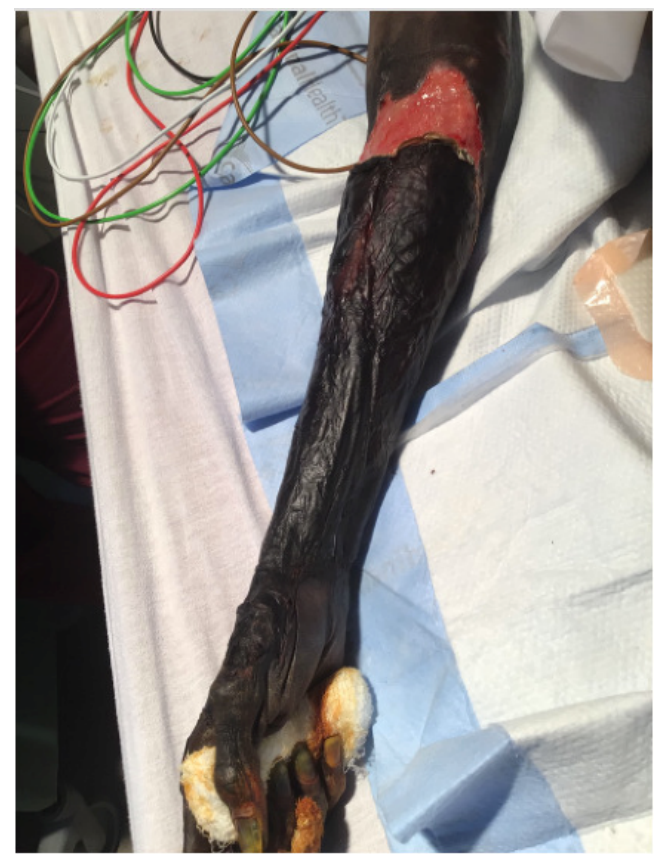

Figure 2. Right upper extremity, post-operative day 53, extensive dry gangrene with underlying viable soft tissue

\section{Discussion}

The case reported herein demonstrates the central role of coagulopathy in the pathophysiology of PF and highlights the importance of supportive care and hematological treatments to reduce the mortality and control the devastating complications of microvascular coagulation and tissue necrosis. Our patient was a previously healthy adult with no known protein $\mathrm{C}$, $\mathrm{S}$ or antithrombinIII deficiencies who developed a polymicrobial abdominal infection and sepsis as the only potential triggers of PF. Her laboratory findings indicated consumption coagulopathy consistent with DIC and she also developed venous thrombosis despite severe thrombocytopenia.

Central to her treatment, we believe, was the initiation of anticoagulation despite her thrombocytopenia, in addition to antibiotics and source control of her systemic infection. Furthermore, the administration of fresh frozen plasma may have contributed to achieving stable protein $\mathrm{C}$ levels, as has been described previously [5]. Elimination of Antibodies by plasmapheresis or immunomodulation with immunoglobulins, steroids or rituximab have also been suggested as potential treatment options $[4,5]$. Due to the short half-life of protein $\mathrm{C}$ in plasma, 2 to 3 units of fresh frozen plasma was recommended for administration every six hours.

Fluid resuscitation is often required in patients with severe sepsis and hypotension. It has also been suggested to improve tissue perfusion through its effect on blood rheology and hemodilution and can therefore potentially be of benefit to patients with PF and microvascular thrombosis. However, our patient suffered from a significant stress cardiomyopathy leading to congestive heart failure and pulmonary edema, limiting her ability to tolerate the recommended volume of fresh frozen plasma. Fluid administration was therefore carefully titrated and balanced with her urine output, which was enhanced using loop diuretics.

\section{Conclusion}

In conclusion, PF is a rare but devastating condition that can lead to limb ischemia and death. Supportive therapy in addition to modern therapeutic options such as steroids, immunomodulation, protein $\mathrm{C}$ zymogen, plasma exchange or plasma therapy can improve outcome and should be considered in the management of these patients.

\section{References}

1. Chambers WN, Holyoke JB, Wilson RF (1952) Purpura fulminans; report of two cases following scarlet fever. $N$ Engl J Med 247: 933-935. [Crossref]

2. Powars DR, Rogers ZR, Patch MJ, McGehee WG, Francis RB, Jr (1987) Purpura fulminans in meningococcemia: association with acquired deficiencies of proteins $\mathrm{C}$ and S. N Engl J Med 317: 571-572. [Crossref]

3. Kizilocak H, Ozdemir N, Dikme G, Koc B, Celkan T (2018) Homozygous protein $\mathrm{C}$ deficiency presenting as neonatal purpura fulminans: management with fresh frozen plasma, low molecular weight heparin and protein C concentrate. J Thromb Thrombolysis 45: 315-318. [Crossref]

4. Edlich RF, Cross CL, Dahlstrom JJ, Long WB (2008) Modern concepts of the diagnosis and treatment of purpura fulminans. J Environ Pathol Toxicol Oncol 27: 191-196. [Crossref]

5. Olivieri M, Huetker S, Kurnik K, Bidlingmaier C, Keil J, et al. (2018) Purpura fulminans-It's not always sepsis. Klin Padiatr 230: 225-226. [Crossref]

Copyright: C2021 Zhu J. This is an open-access article distributed under the terms of the Creative Commons Attribution License, which permits unrestricted use, distribution, and reproduction in any medium, provided the original author and source are credited. 